\title{
Virtual reality in manufacturing: immersive and collaborative artificial-reality in design of human-robot workspace
}

\author{
Ali Ahmad Malik, Tariq Masood and Arne Bilberg
}

University of Southern Denmark and University of Cambridge
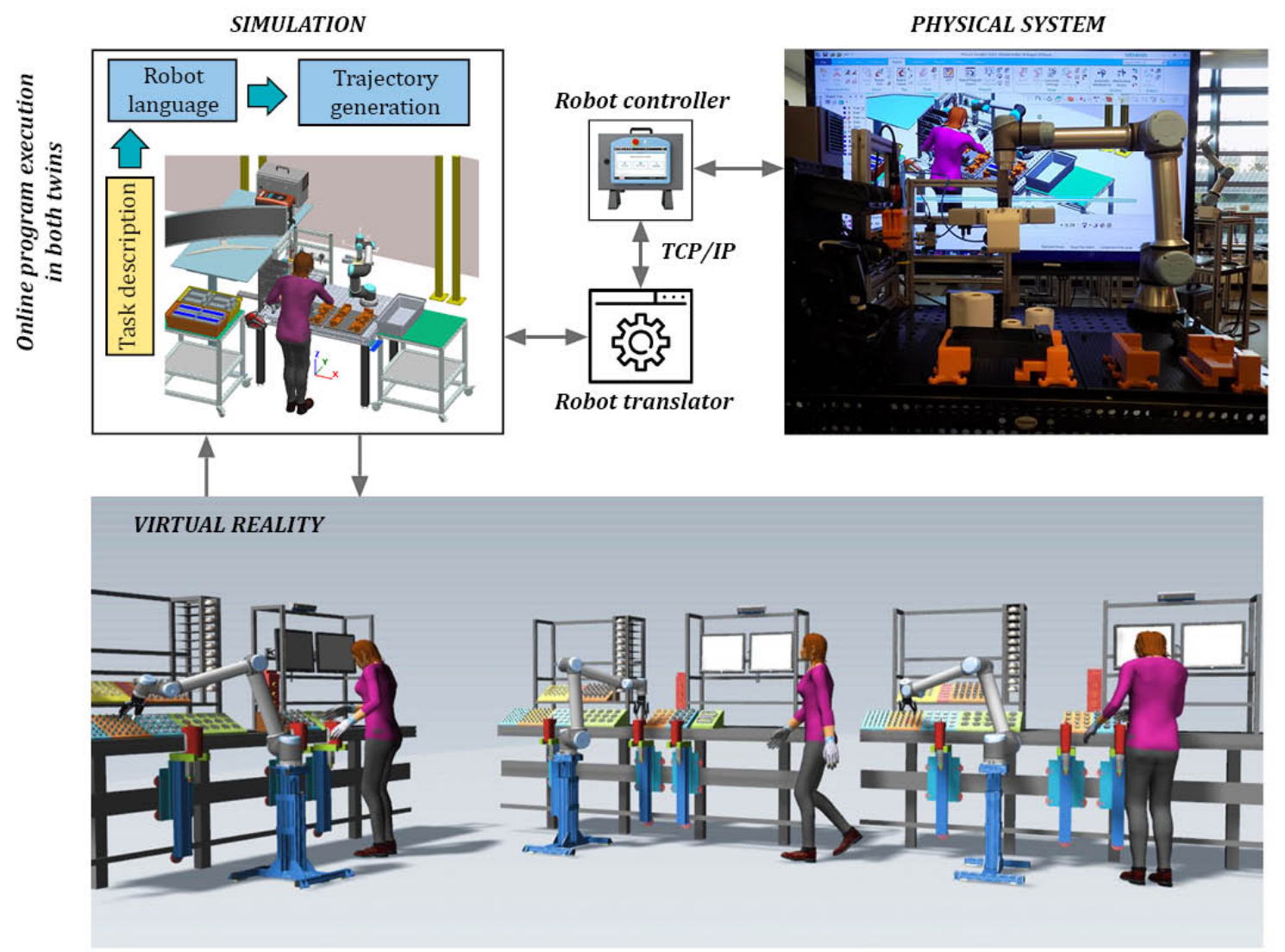

\section{Highlights}

- This paper explores the technological development in virtual reality for design of human-centred production systems and develop a unified framework to combine human-robot simulation with virtual reality. 


\title{
Virtual reality in manufacturing: immersive and collaborative artificial-reality in design of human-robot workspace
}

\author{
Ali Ahmad Malik ${ }^{*}$, Tariq Masood ${ }^{2}$ and Arne Bilberg ${ }^{1}$ \\ ${ }^{1}$ Mads Clausen Institute, University of Southern Denmark, Sønderborg, Denmark \\ ${ }^{2}$ Department of Engineering, University of Cambridge, 17 Charles Babbage Road, \\ Cambridge CB3 0FS, UK \\ *E: ali.malik@mci.sdu.dk
}

\begin{abstract}
Over the past years the concept of human centred automation has received a lot of attention to achieve hybrid automation. A form of hybrid automation is taking benefit from the synergistic effect of human-robot collaboration. When used in assembly, the requirement of flexibility, adaptability and safety makes the design and redesign of human-robot collaborative (HRC) systems a complex and prone to error process. The use of time-based continuous simulations can offer safe virtual space for testing and validation thus easing the design of complex HRC systems. However, conventional simulations don't allow to experience the future production system as an end-user in an immersive environment. This paper explores the technological development in VR for design of human-centered production systems and develop a unified framework to combine human-robot simulation with VR. The simulation as an event-driven simulation helped in estimating the humanrobot cycle times, developing process plan, layout optimization and robot control program. The same simulation is used in VR to interact with the production equipment and particularly with the robot. Additionally, AWS Sumerian environment is used to create a virtual robot to assist VR user in the design process.
\end{abstract}

Keywords: Simulation; human-robot collaboration; virtual reality; VR; Cobot; Digital twin

\section{Introduction}

Virtual reality or VR is a digital artificial environment that makes the human-senses to perceive and experience it as real. The significant development in visualization technologies in the past years has shaped the next wave of VR with high resolution 
displays and interactive input devices at affordable prices (Anthes et al. 2016). These technologies are including immersive virtual reality headsets, motion-tracking hardware and high-resolution graphics ( $\mathrm{Li}$ et al. 2018). However, the aforementioned technological development is greatly being driven by enthusiasts having interest in VR rather than established scientific community (Anthes et al. 2016). As suggested by (Anthes et al. 2016) the creation of knowledge in any new technology in the early diffusion stage may act as a catalyst for rapid diffusion by increasing the probability of success. This requires that the technological development is explored in many different areas for possible advantages.

The usefulness of virtual reality for design and redesign of complex manufacturing systems has been discussed and encouraged in various studies (Kuehn, 2006; Novák-Marcinvcin, 2007; Brettel et al. 2014). The concept lies in developing a three dimensional virtual or digital model of the manufacturing system that represents the properties of the real system. The real system may be a new system being developed or an older system being optimized. VR enables the engineers and technologists to experiment the system in an intuitive and close-to-reality virtual environment, well before making any investment thus decreasing the probability of failures (Rauschnabel, Brem and Ro 2015).

With the growing demand of customization and flexibility, modern day manufacturing systems are getting increasingly complex (Bilberg and Malik 2019). The attention towards integrating flexibility of humans and efficiency of machines is making humans the central part of a manufacturing system (Flemisch et al. 2012)(Malik and Bilberg 2019a) thus transforming the manufacturing system from being mechatronic nature to biomechatronic architecture. 
Human-robot collaboration (HRC) is one example of the dream scenario of humanrobot work teams sharing their workload. HRC systems are denoted as complex systems due to their requirement of being adaptable and safe for fellow human(s). The challenges associated with human-robot collaboration (Hayes and Scassellati 2013; Tsarouchi, Makris and Chryssolouris 2016; Malik and Bilberg 2017) are keeping its industrial application in a high-level of human-robot engagement in its infancy. For designing an HRC workstation the major challenges are skill-based tasks distribution between robot and human, quick adaption and validation of workstation layout, virtual commissioning of the designed production system and ensuring safe working conditions for fellow human worker(s).

This paper aims at exploring the usefulness of VR for fast validation of the HRC workspace. It is argued, through a case study and experimentation, that the combined effect of digital human-robot modelling and immersive and collaborative VR can make the design and re-design of HRC systems fast and reliable. The contributions of this paper are:

- Presenting a structured framework for using VR in design of HRC workspace

- Linking of a real physical robot with VR and event-driven simulation to intuitively and online program the robot

- Using a virtual chatbot as an assistant in the VR environment

\subsection{Human-robot collaboration}

Assembly in manufacturing accounts for handling complex part geometries, high number of product variants and fluctuations in production orders (Hu et al. 2011). Assembly systems need to be responsive and flexible to deal with above conditions. Such 
manufacturing flexibility has traditionally been achieved by humans (Müller, Vette and Scholer 2016). However, manual systems are not able to effectively response to the challenges of globalization and the growing need of mass customization (Takata and Hirano 2011).

Conventional automation solutions have achieved tremendous success in the past decades and is greatly attributed towards industrial robots, but these solutions are rigid and lack the flexibility required for being responsive to fluctuating demands. Therefore assembly, as an example of human intensive work, has traditionally been remained away from physical automation.

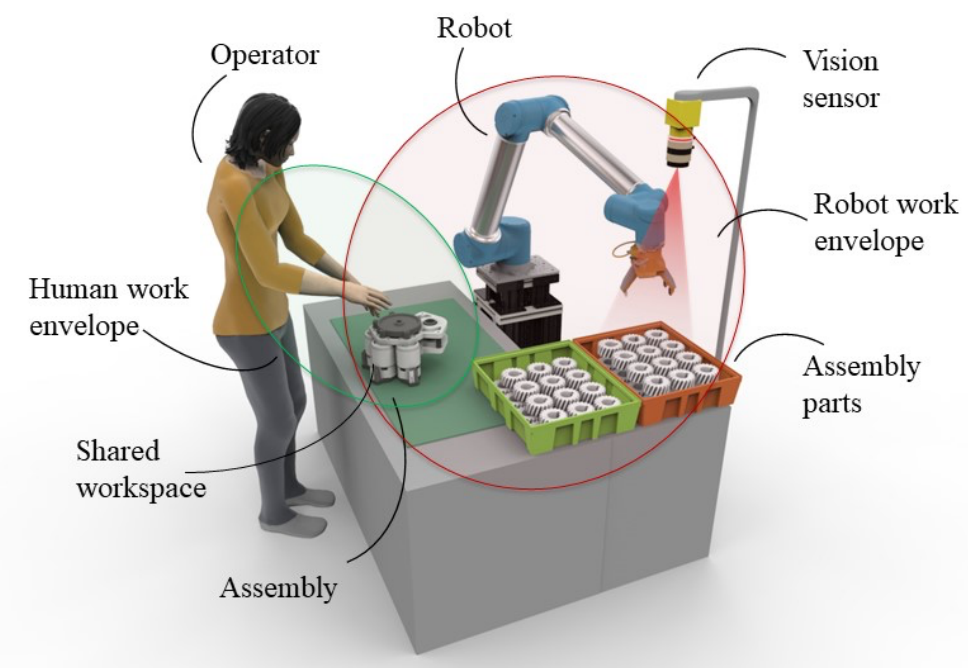

Figure 1: Human robot collaboration (Malik and Bilberg 2019b)

The term of human-centred-automation was introduced by Billings (Billings, 1997) from the domain of aviation sciences as an approach to system design, "in which humans and machines collaborate cooperatively in order to reach stated objectives". A hybrid combination of human and machine, by taking benefit from the synergistic effect of human-machine collaboration (Figure1), can potentially offer an increased productivity, 
higher product mix and production reconfiguration. The concept is aligned with Lean Automation (Jackson et al. 2011; Bilberg and Hadar 2012; Mrugalska and Wyrwicka 2017) to develop flexible manufacturing structures. The core of the process is on the argument that humans and machines both have strengths and weaknesses. By keeping humans as the core of the automation process the required and right automation level is attained. Thereby achieving a production structure of having worker and machine tightly bound together taking benefit from each other's strengths and compensating for each other's' weaknesses (Krüger et al. 2017).

One way of enabling human-machine collaboration is through the new generation of industrial robots or Robotics 2.0. The new paradigm of industrial robots are humanfriendly robots, referred to as collaborative robots or cobots. The flexibility and dexterity of a cobot is inherited by the variety of manageable tools, sensors, supporting devices. The kinematical degrees of freedom allow a cobot to reach every coordinate of its workspace in multiple configurations (Djuric, Urbanic and Rickli 2016; Michniewicz and Reinhart 2016). Together with advanced safety devices and protocols the cobots are enabling workspaces with right amount of automation (Müller, Vette and Scholer 2016) developing a balance between flexibility of manual processes and repeatability of machines (Thomas et al. 2016). 


\subsection{Virtual reality in manufacturing}

The interaction of humans and robots in industrial environment is seen as a complex system. Although there is not agreed definition for complexity but the information content, many to many interactions, and hard to predict the behaviour denotes its complexity (Grieves, 2019).
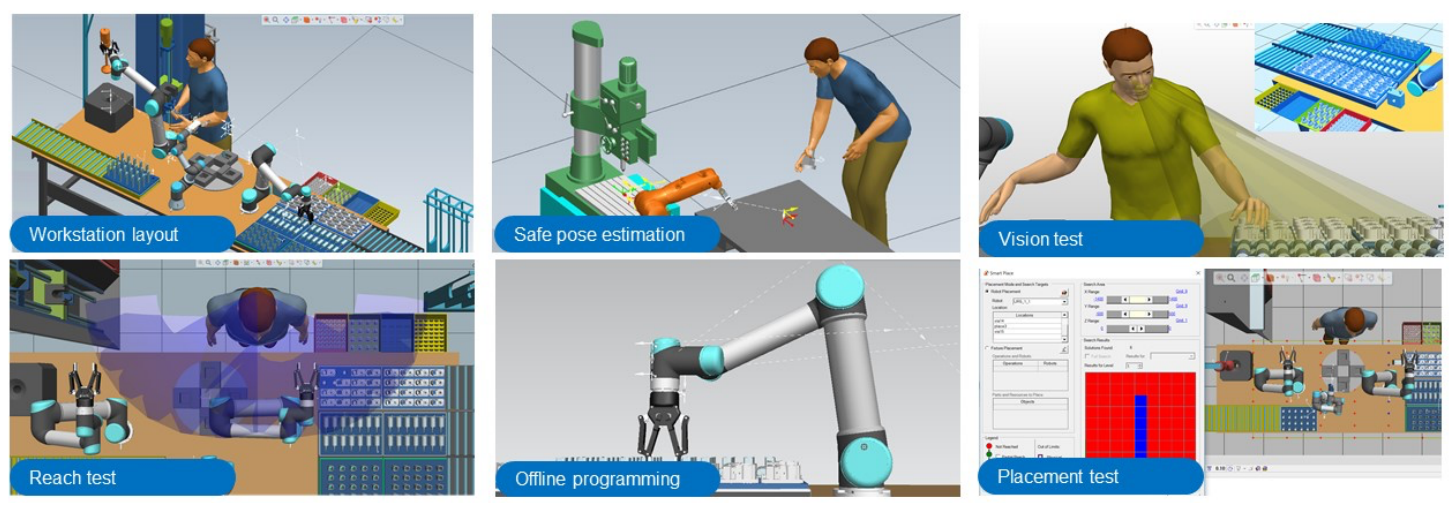

Figure 2: Simulation in design of HRC systems (Malik and Bilberg 2018)

A way to design and validate complex systems is through enabling virtual spaces (Figure 2) as investigative arenas. Computer aided design (CAD) and simulation tools have been used successfully to offer fail-safe virtual environments (Figure 3). These tools offer decision makers and engineers to develop close-to-reality 3-dimensional digital models of the production equipment with their dynamic behaviour to make low-cost, 


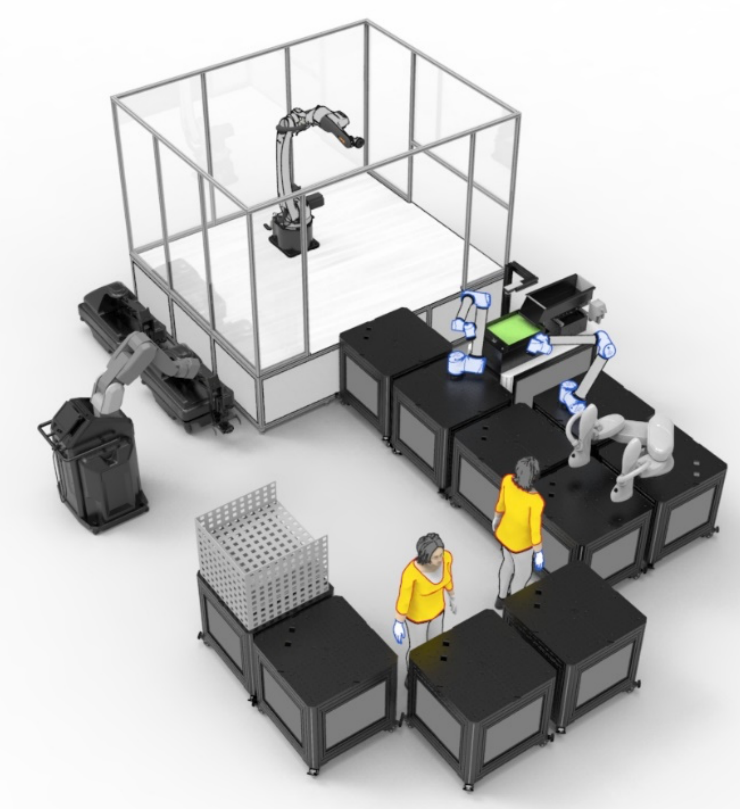

Figure 3: Virtual simulation in design of a production system

Once the design-decisions are finalized the robots used in HRC are programmed either by online robot programming tools (i.e. teach pendant) and offline programming tools (i.e. graphical software). Offline programming tools are both brand specific (e.g. Robot Studio, KUKA) and some are general purpose (Robot Expert, Process Simulate, DELMIA). When it comes to digital modelling of human work-tasks, the simulation of digital human model has been a challenging task and is often not integrated in manufacturing virtual simulations. If used, the quality of the digital human model is far from reality. This keeps an important aspect of a production system (i.e. human) out of the loop in the planning phase. Most often humans, as end users, only evaluate the new manufacturing system in a physical-simulation late when development work is done.

The research of combined human robot simulation for production analysis is limited and often researchers have argued that limited literature has discussed software able to 
simultaneously simulate human and robot collaboration (Ore, Hansson and Wiktorsson 2017).

The possible advantages of HRC are evident from literature but due to the challenges the technology is still in its early phases of maturity for industrial application while the reliable methods of safely, quickly and intuitively validating these systems are very limited.

\subsection{Immersive and non-immersive virtual reality (VR)}

Loeffler (Korves and Loftus 1999) had described VR as, "a three-dimensional, computer generated, simulated environment that is rendered in real-time according to the behaviour of the user". The objective with VR environment is to develop maximum sense of presence between the user and the computer. Although the modern day use of the word VR is linked only with immersive VR however, the VR environments can be characterized as non-immersive and immersive VRs. Non-immersive VR systems enable the user to visualize the digital models on a screen (e.g. desktop systems and widescreen projections) while immersive VR systems are often visualized with a head-mounted display that let the user virtually 'enter' into the scene and interact with the virtual models (e.g. immersive CAVE systems and immersive HMDs) (Korves and Loftus 1999).

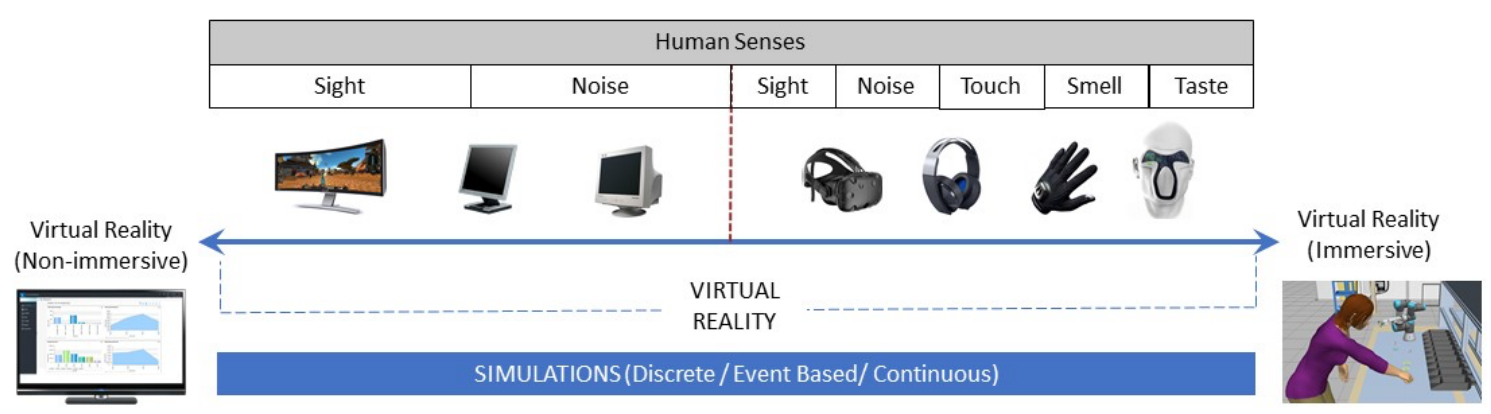

Figure 4: Immersive and non-immersive VR in connection with manufacturing application

A representation of various level of virtual reality as used in manufacturing is 
given in Figure 4. The conventional (non-immersive) VR is on the left side. In this case, the visualization is available on a computer screen that triggers the human-senses of sight and hearing during VR experience but keeps the user attached to the real world making the user aware of what is going around e.g. sound, visuals and haptic. The immersive VR (Figure 5) is on the right side that can also trigger the sense of sight but isolates the user from the real world and enables the user to interact with the VR environment using special haptic devices. Several studies have documented the relationship between the screen size and viewers' sense of being tin the simulated scene (Simon and Greitemeyer 2019). Additionally, the human senses of touch (Bar-Cohen, 2003), smell, and taste (Ranasinghe et al. 2014) in addition to the sight and 3D sound (Begault and Trejo 2000) are being explored by the research community. The modern day use of the term VR is associated with immersive-VR (Liu et al. 2017; Michalos et al. 2018; Liu et al. 2017) however, the virtual simulation visualized on a computer screen is also a VR technology but is of nonimmersive nature. A measure to immersion is the feeling of presence (Simon and Greitemeyer 2019). However, this study is aimed to signify the value of immersive VR in human-robot simulations besides advantages of conventional non-immersive VR.

A renaissance is being experienced in the research, development and use of HMDs for VR. With significant price reductions, the VR devices are gradually getting accessible by the smaller companies and hobbyists. A challenge associated with developing high quality VR experience has been the low computational power of the devices, required for the refresh rate (needed to smoothly translate head movement in the virtual space), resulting in inadequate resolution coupled with a low field-of-view (Hilfert and König 
2016). This challenge has now been solved by the ever-increasing computational power of computers.

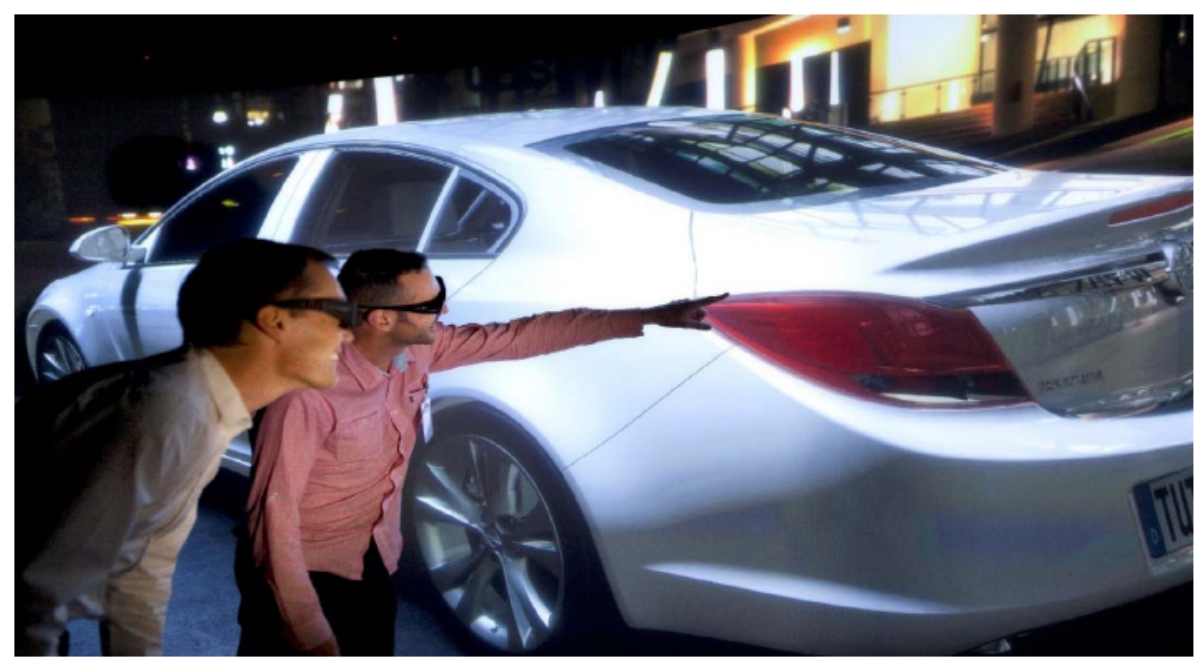

Figure 5: Immersive VR in practice (Agrawal 2018)

Like for any complex system, VR in design of human-robot collaboration, can make the user to explore the environment offering an intuitive way to identify design issues and optimize the HRC strategy. In an HRC-VR, the user can guide the robot arm by virtually interacting it, make changes in the layout by using handheld controllers, make dimensional assessments for layout optimization and run the dynamic simulation. Thereby, the three-dimensional VR visualization makes the production system better understandable for the end-users. An important feature is that all the changes made within immersive VR experience are translated to the simulation model that can generate robot program for the physical robot.

\section{Related work}

Acquiring of information to predict the behaviour of any complex system or process is useful to make the process error proof before it goes into operation (Pelliccia 
et al. 2018). A method for HRC workplace design and task planning was described by (Tsarouchi et al. 2017) that also comprised virtual facility layout and evaluation of alternative designs. Both human and robot were modelled in a unified simulation and the model is used for task allocation problem. However, immersive VR visualization was not a part of this study. A simulation based task allocation approach between mobile robots was discussed by (Michalos et al. 2014). In this study a semantic model representing the robot and operations was used to assign the tasks to the appropriate robot and generate robot paths.

$\mathrm{VR}$, as an investigative tool, has been documented in the design and validation process of complex systems (Muhanna, 2015). Considering the complexities of production environment, the field of robotics and especially collaborative robotics have received considerable attention from the VR research community. An approach to link a robot with VR to evaluate the performance of a robotic gripper has been presented by (Pelliccia et al. 2018). An early stage demonstrator to highlight the value of robot programming via VR was discussed by (de Giorgio et al. 2017) using Unity game engine. The use of VR for hip prosthesis implantation using a robot simulator was documented by (Kaluschke et al. 2018).

An emerging trend in design and operation of complex systems is a digital twin. The digital twin is a virtual representation of elements and dynamic of a physical system that supports the prediction and operation of the physical system throughout its lifecycle. Combining a digital twin and VR to design the manufacturing system for visualization and virtual commissioning utilizes the digital cope for VR visualization. An architecture was also proposed combining virtual prototype in design of cyber physical systems.

A recent work on workplace analysis and design using virtual reality was presented by (Michalos et al. 2018). The writer used a "Spaghetti Chart" as a lean tool 
for decision making during support to assembly workstation layout design. An immersive CAVE system was used to estimate operation completion time, travelled distance and ergonomics. However, the system was designed as a virtual animation where the robot kinematics and digital human simulation were out of scope. This simulation study was a standalone VR experience

The mentioned studies are not highlighting the need and value of VR in a systematic HRC design process. A systematic design process for an HRC system combining simulation and VR with a digital twin approach is needed. A unified simulation as part of a larger framework can have interlinked benefits for many design and development activities.

\section{Virtual reality framework for HRC design}

This section presents a framework (Figure 6) for designing HRC workplace combining simulation and VR. Designing a production system is a systematic, goaloriented and structured design process starting from the idea to the start of production. A systematic approach to an iterative and systematic engineering design is Roozenberg's Engineering Design Cycle. Based on this cycle, the first element (ELEMENT-1) of the VR-framework describes the steps when designing an HRC system (Malik and Bilberg 2017). The design of the product and its details initiate the process, requirements are documented and analysed and in the synthesis phase, the design of the HRC system is carried out. The design variables for designing HRC system are suggested by Ore (Ore, Hansson and Wiktorsson 2017) as robot type, robot position, gripper design, material position, workstation equipment and humans. Roozenberg emphasized the need of 
simulations for testing and analysis to remove unpredicted undesirables in the early phase.
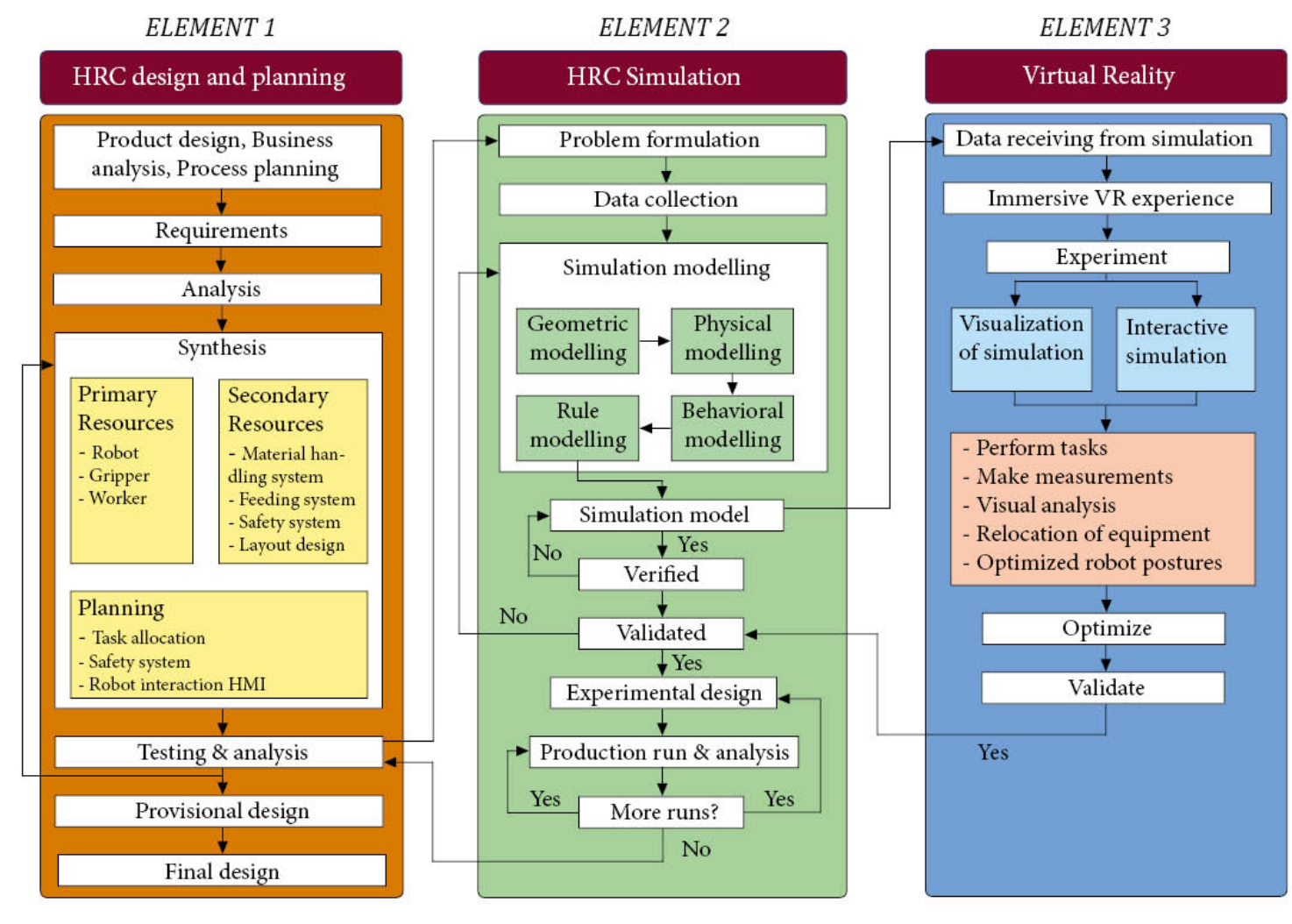

Figure 6: Framework for unifying simulation and VR for HRC design and planning

The ELEMENT-2 is the simulation environment for test and validation. The purpose to use simulation is to identify the errors and reach the final design in shorter time and less effort. The simulation process is a repetitive process of (Bilberg and Alting 1991) modelling, analysing and optimising (Bilberg and Alting 1991). All the production assets necessary for simulation and corresponding to the elements of the physical system are created as $3 \mathrm{D}$ CAD data. The virtual robot model is designed with the inverse kinematics and is using the robot controller provided by the robot manufacturer. The digital human model is integrated in the simulation model. To make human movements more realistic an RGB-D (Red, Green, Blue \& Depth) camera for motion recording is used for capturing motions of a real human. These RGBD sensors can work both as (a) Realtime in simulation model: in this case it helps to determine the ergonomics of a real 
operator; (b) to record the human movements and use later in the simulation. A factor to be considered is that the virtual model must be kept simple and may not be of the equal complexity as the physical system.

At this stage the assembly sequence is also modelled following the assembly precedent constraints and the skills of human and robot (Malik and Bilberg 2019b). The assigned tasks are modelled in the simulation by defining the key locations for picking and placing the components. Adjustments of the robot motions maybe required for pick and place locations both for robot and human. With several iterations, the simulation is achieved representing the real production system. The result from this stage is an initial layout of the workplace, work task sequence and estimated cycle times.

In the simulation modelling phase, the precision and accurate interpretation of the simulation results define the authenticity of the whole design and development process. At this stage, the use of VR can help the designer and end-user to get into the scene and make realistic evaluations. The end-user can experience the scene in almost as final version thus enabling better and faster evaluation.

Thereby, the ELEMENT-3 defined the process of utilizing VR capability to mature the design. After this the digital model can be experienced with a head-mounted display for immersive VR experience. The immersive effect of visual and haptic VR assists the user to intuitively visualize the model and its dynamic simulation and thereby deduce results that help to finalize the HRC effectively. The interlinking of the VR process with the simulation and finally to the HRC design cycle reduced the design and development time.

The optimized model is then used to define the bill-of-processes (BOP) and production equipment required. When connected with the real robot, once the simulation 
is completed, the robot codes are generated and are downloaded to the physical robot for further experimentation.

\section{Experimental setup}

\subsection{HRC system}

The production setup used for testing and demonstration is an assembly workstation where a human and a robot are working together. The setup has been investigated for industrial production of battery packs that need to be used with electronic linear-actuators in hospital beds. The production cell consists of a collaborative robot namely UR-5 developed by Universal Robots. The UR-5 robot has 6 degrees of freedom (DOF), a payload capacity of $5 \mathrm{Kg}$ and a maximum reach of $800 \mathrm{~mm}$. The gripper used with the robot is a SCHUNK parallel gripper. The robot is used to take a portion of the total tasks of the assembly process. The remaining tasks are carried out by a human operator. The human operator in this case is a female with height of $172 \mathrm{~cm}$ and body mass index $(\mathrm{BMI})$ of $<25$. The fixture required to hold the product during $\mathrm{HRC}$ assembly are made as 3D printing. The fixture is needed both for the operator and the robotic tasks.

\subsection{Developing the virtual-simulation model}

The virtual simulation model is representing the above-mentioned assembly workstation. The virtual model is created in a CAD software named Siemens NX. The virtual model consists of both active objects and passive objects. The active objects represent those objects that are directly used in the production e.g. humans, robots, machines, etc. while passive objects are not directly used in production e.g. safety fences, tables etc. Both the active and passive equipment in the virtual production system are then saved in JT (Jupiter Tessellation), which is a 'lightweight' 3D data format. This data is then imported into a continuous simulation tool i.e., Tecnomatix Process Simulate (TPS) 
(Figure 7). The selection of the tool is entirely based on availability and comparison of its capabilities to other similar software is beyond the scope of this study.

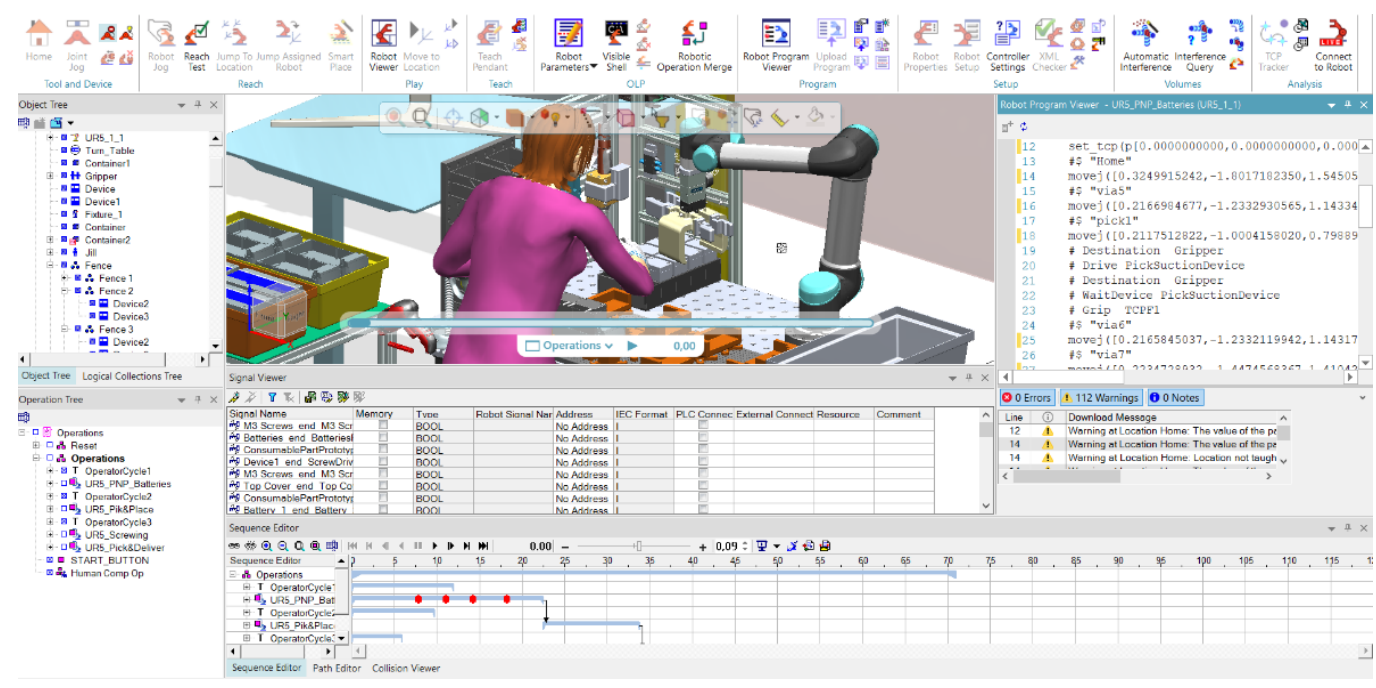

Figure 7: The virtual model of the HRC system in event-driven simulation

The human object is developed as a three-dimensional deformable object. Various human postures (Figure 8) are combined to build the simulation of human tasks e.g. pick and place a product. Each task is divided into elementary phases (e.g. Go, Get, Put, Position) and by compiling all these together a holistic simulation of the human task is formed (Figure 7). The skin of the human object is represented as a single deformable mesh that updates dynamically in real time as the virtual human is manipulated for a realistic appearance.

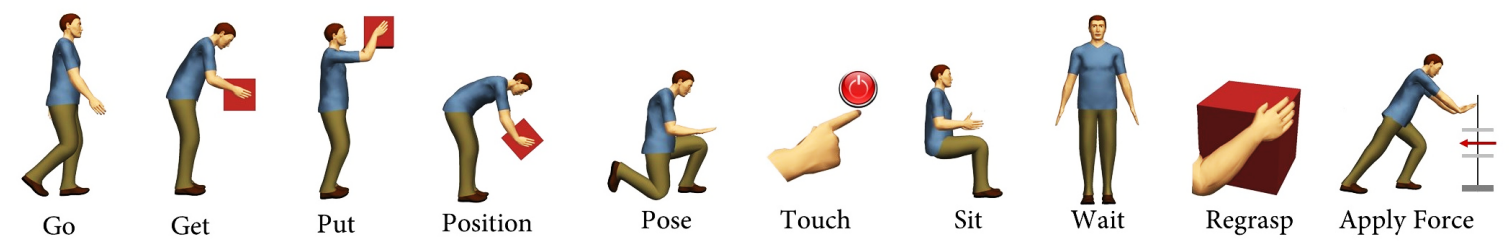

Figure 8: Elements of human tasks in a virtual model 


\subsection{VR equipment}

Several HMDs based on varying technologies are available in the market for immersive VR experience. For this research, HTC Vive is used that consists of one HMD and two handheld controllers (Figure 9). During use, the HMD displays two different images for the right and left eyes. The images are slightly shifted relative to each other to make a stereoscopic image (Dahl et al. 2017). The HMD and the controllers are equipped with several light sensors for orientation and position tracking in the room.

For this purpose, two laser emitters are installed in each corner of the room (Figure 9). To enable uninterrupted tracking of the user while moving freely in the VR space the laser emitters emit an arc of laser light in sweeping pattern. Multiple spatial distributed photo diodes measure the time taken to activate the photo sensor. This help the headset to track the exact position in the VR space. The same technology is applied for the hand controllers that assist in grabbing and manipulating objects.
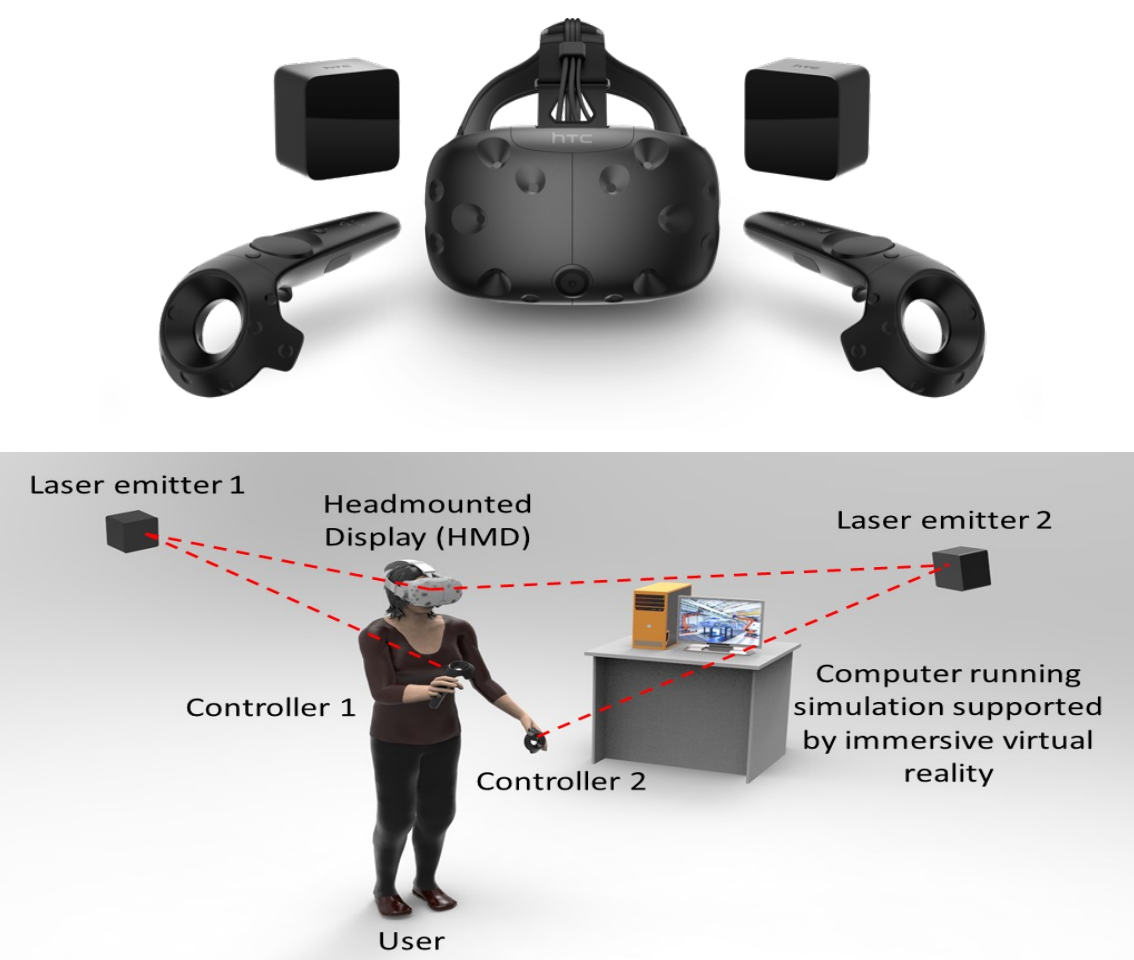

Figure 9: VR hardware and its room setup

Citation details: Malik, A.A., Masood, T. and Bilberg, A. (2020) 'Virtual reality in manufacturing: immersive and collaborative artificial-reality in design of human-robot workspace', International Journal of Computer Integrated Manufacturing, 33:1, 22-37, https://doi.org/10.1080/0951192X.2019.1690685 (accepted 4 November 2019). Page | 18 


\subsection{Motion and posture tracking}

To make realistic human posturing a virtual reality motion tracking device (Microsoft Kinect) is used. This helps to drive the human model in the scene. Motion tracking gives realistic insights to how people perform a task or interact with a product design. The software also helped to record all the human motions and integrate them later in the simulation (Figure 10). This step is optional and is not mandatory. The human motions can either be generated with predefined human posture blocks or by using Kinect sensor for scanning the positions and postures.
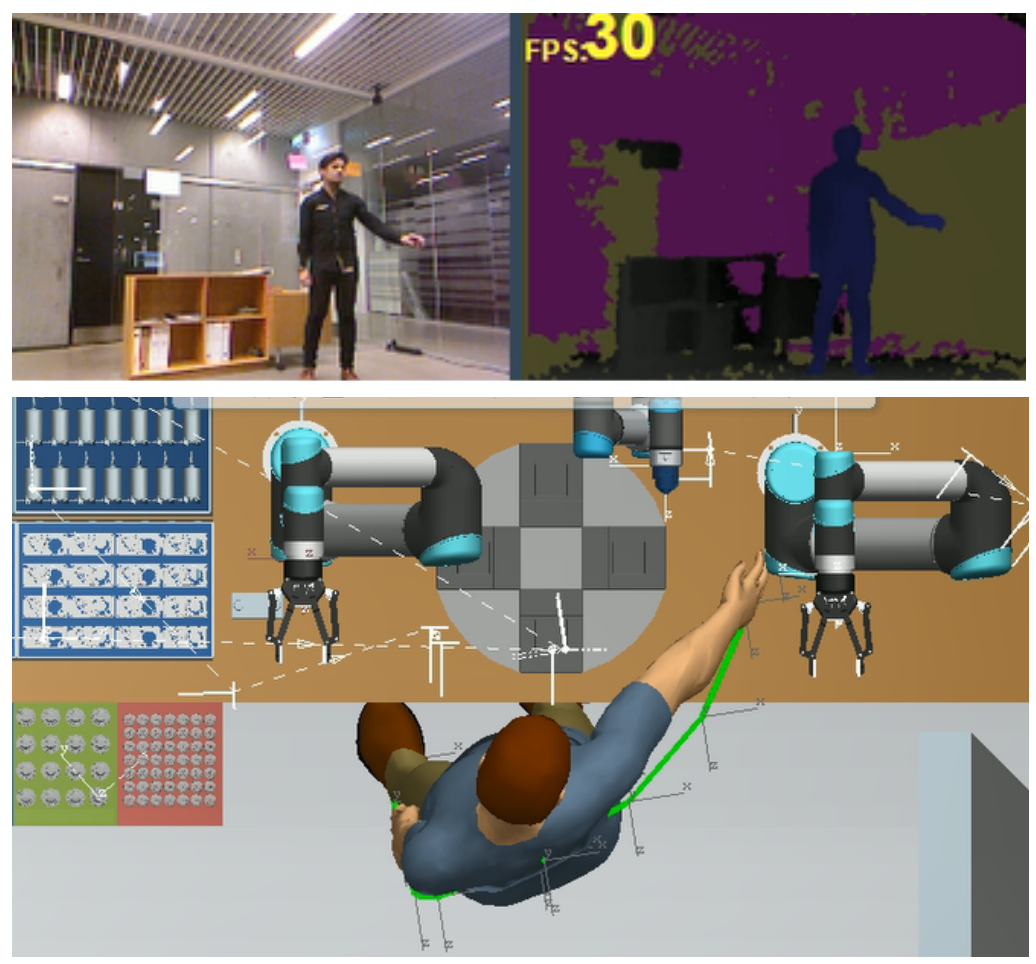

Figure 10: Kinect sensor for accurate human-posture tracking

\subsection{The VR environment}

Finally, the virtual model is viewable in the VR hardware (HMD). The VR hardware is activated using Steam gaming platform. The simulation is also real time synced with a real robot (Figure 15). The connection between a real robot, a 
simulation environment and VR environment enable the users to experience the HRC cell at a full factory level and also to program the robot with interactive manipulation.

\section{Results}

This chapter presents the results achieved from the experimentation. The intuitive and immersive visualization in itself is a way to better understand the system and eventually form better and faster designs. Therefore, the immersive visualization is the first thing to achieve with VR application.

\subsection{Immersive realistic visualization}

In the VR environment, the user can move around in the virtual space either by walking physically or by teleporting the self without any physical movement. For the later, two hand-held controllers are used to generate teleporting instructions to the VR controller. The same controllers are used to interact with the virtual equipment and relocation of the equipment in the virtual space. This enables the users to test the production system and robot actions, as being in real.

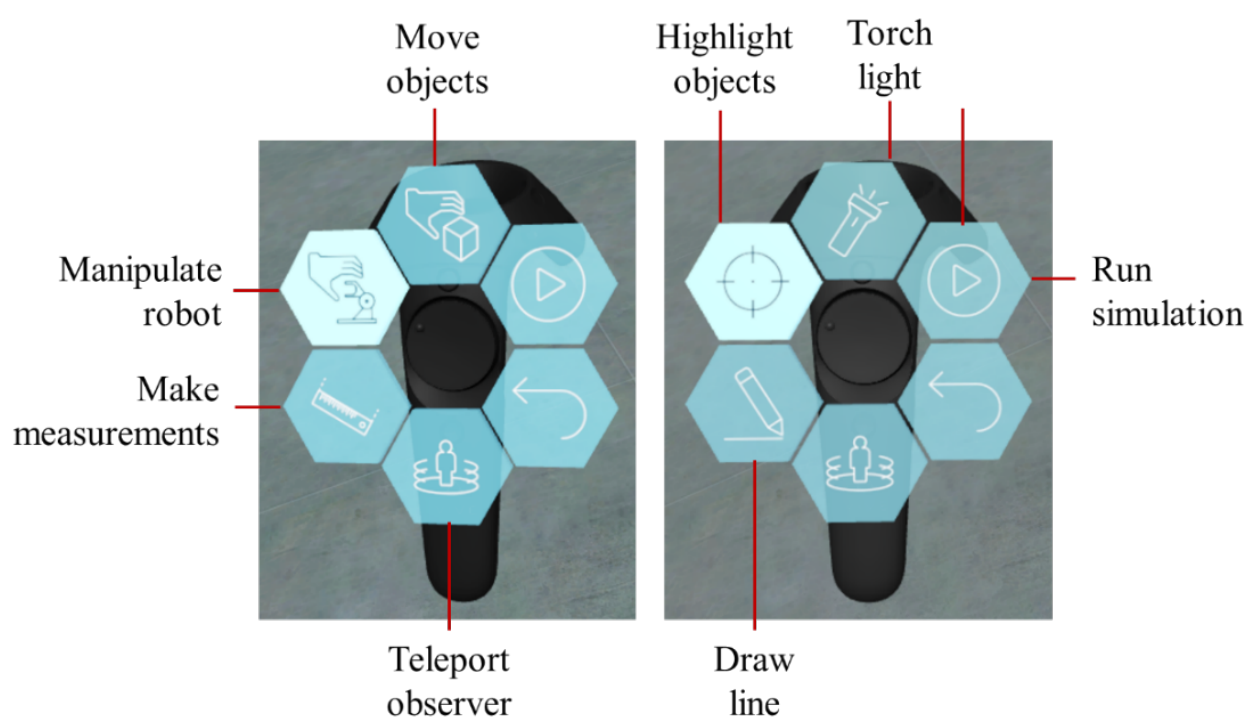

Figure 11: Controllers for immersive VR environment 
Using the control buttons, at the hand-held controllers, the simulation is started by the user. The user examines the dynamic simulation while being in the VR scene and makes a pause and restarts the simulation as and when needed. The user can examine the placement of HRC objects by approaching to them and visualizing them in different angles just like in the real world (Figure 12). If an object needs repositioning, the object can be picked and replaced or re-oriented by using hand controllers. Since the human model is now at the actual head-height of the user, the user can examine the robot movements in a realistic way and can examine if the robot reaches at or above the human head height, increasing safety risks for human.

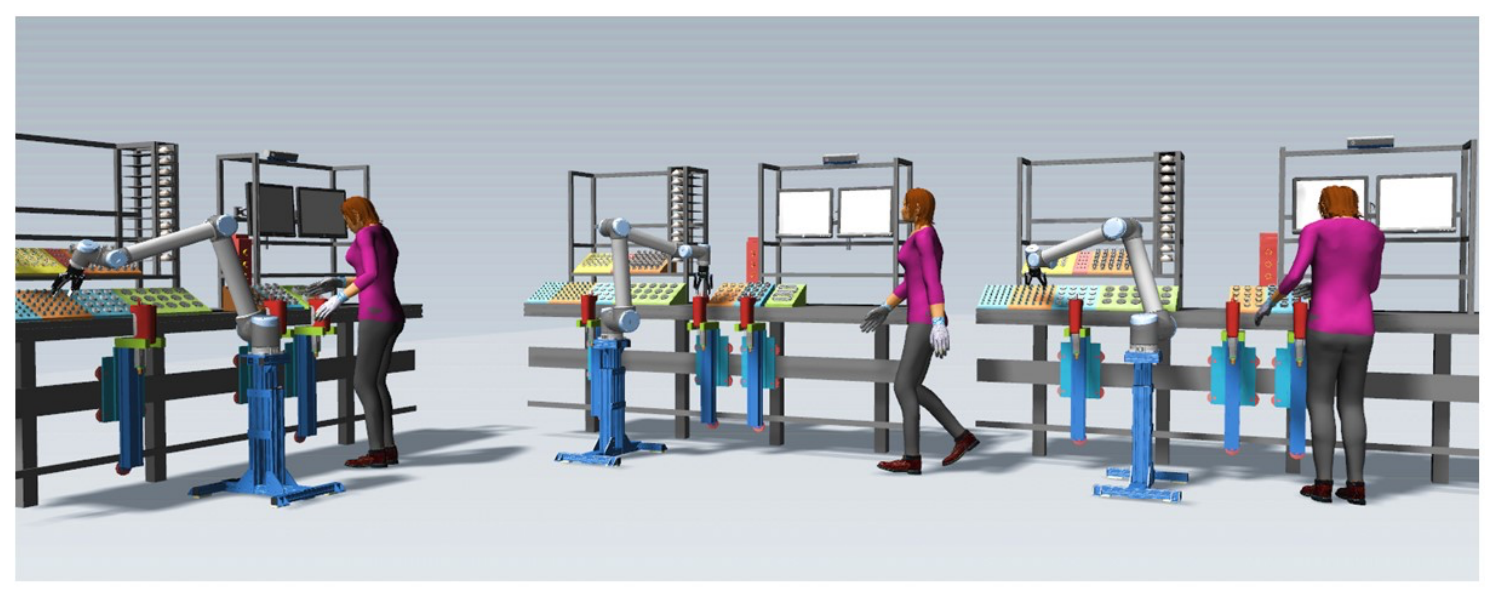

Figure 12: Event-based simulation and VR environment of a factory floor

The robot is integrated with joint kinematics in the simulation model. To visualize the movement and reachability of robot, the user can interact with the robot from its endeffector and can move it to different positions while maintaining all its joint constraints (Figure 13). If the robot arm is taken out of the reach of the robot a haptic vibration is initiated in the hand controller, informing the user that the object being accessed is out of the robot reach. Robot manipulation lets the user to move it to a new position thus defining a new robot pose and path. The (re)defined robot position will be transferred to the 
simulation study in real-time and later robot codes may be generated. All the placements of the equipment can be examined with measurement tools to measure the distances between objects (Figure 13). If a distance is observed to be readjusted the object is picked and repositioned. Since the data being visualized in VR is directly connected with the virtual event-based simulation therefore all the changes defined during VR experience are transferred to the simulation.
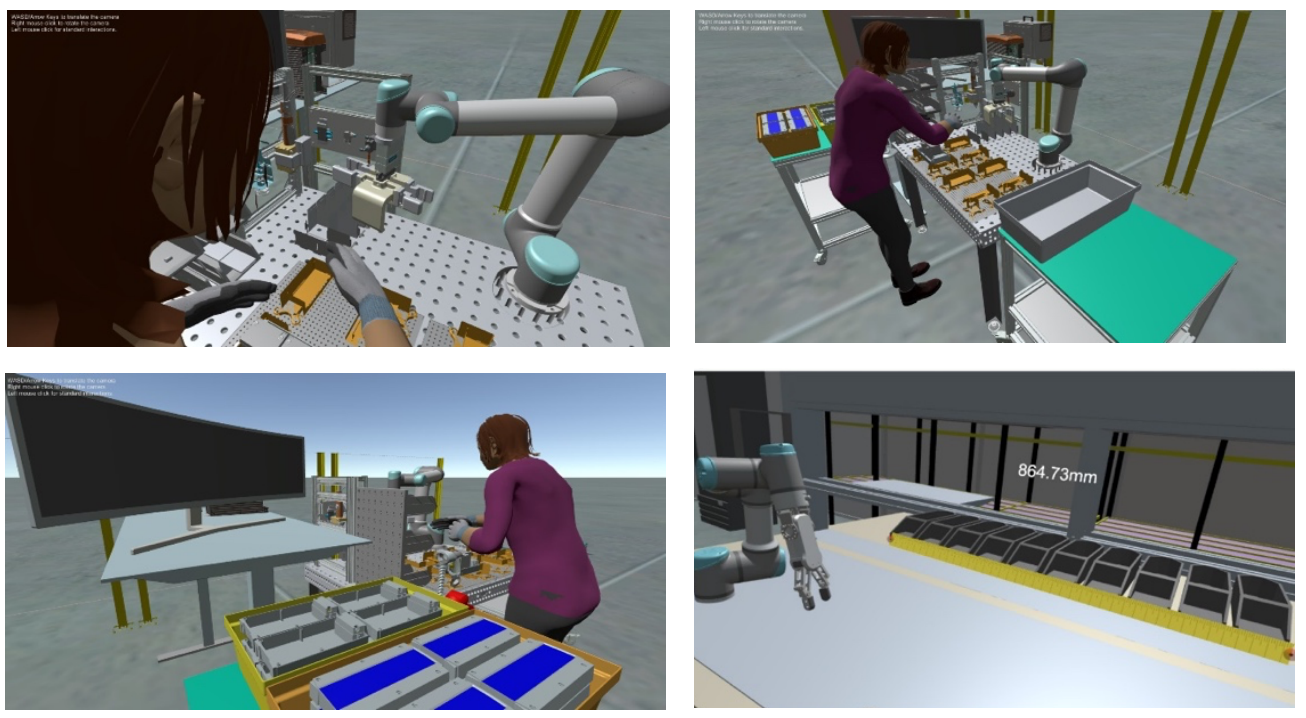

Figure 13: Analysis in VR by the user
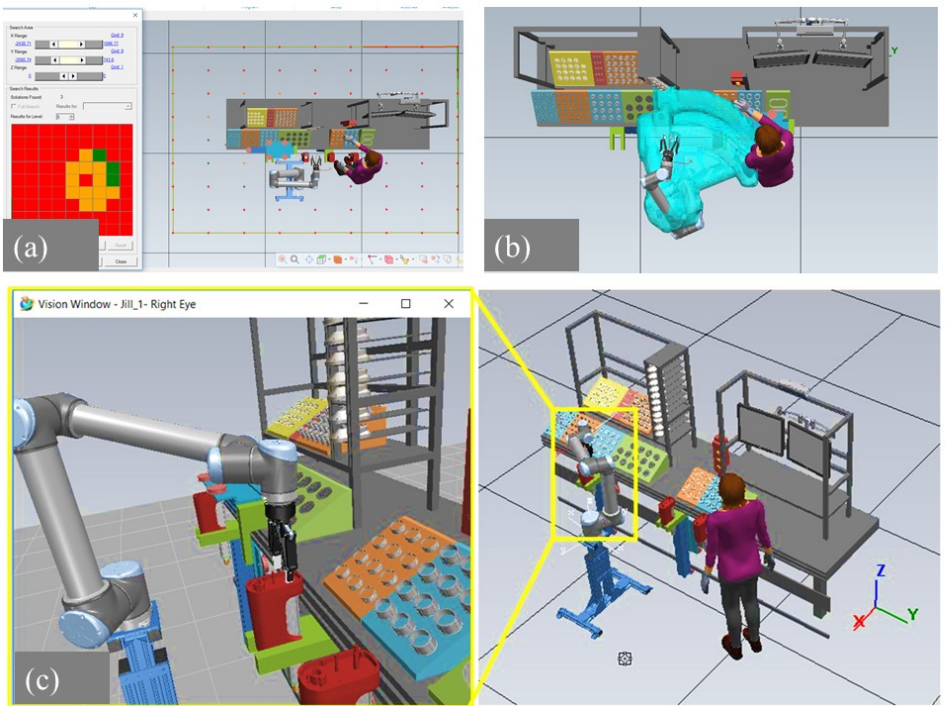

Figure 14: (1) Placement test in simulation for HRC planning; (b) Collision analysis between human and robot; (c) Vision analysis through human's eye in the simulation

Citation details: Malik, A.A., Masood, T. and Bilberg, A. (2020) 'Virtual reality in manufacturing: immersive and collaborative artificial-reality in design of human-robot workspace', International Journal of Computer Integrated Manufacturing, 33:1, 22-37, https://doi.org/10.1080/0951192X.2019.1690685 (accepted 4 November 2019). Page | 22 
The optimal placement of robot, human and other equipment is of utmost importance for collaboration and shorter cycle times. The desired safety level also gets increased because of the co-existence of humans and robots in the same workspace. The following sections describe the steps to achieve a better workstation layout:

(a) Collision analysis: Collisions are likely to occur in an HRC system with human and robot entering each other's workspace. The simulation helps to detect possible collisions and suggests optimization for robot trajectories or relocation of the robot or equipment for equipment related collisions. An important feature of cobots is to halt in case of or before (near miss) a collision and as soon the human leaves the workspace the robot starts the production cycle. However, frequent collisions tend to reduce the productivity and therefore must be avoided.

(b) Reach test: Reach test determines the reachability of a cobot and human in all desired locations within their workspaces and thereby determines the most optimal placement locations for cobots and the equipment. In case of a human, the task is achieved by creating a virtual grasp-envelope to assess reachability of human arms without bending the body beyond safe limits.

(c) Placement test: Defining optimal placement locations is necessary to minimize cycle times and develop safe working conditions. Placement test achieves the goal by evaluating the layout and defining a set of points from where the cobot can reach its desired pick/place locations and work coordinates.

(d) Vision test: This test enables to open a window showing the view from the human's eye working in alongside the robot. By viewing the workstation through the eyes of the 
digital human model, the possible obstacles in eye-sight can be examined and optimized.

\subsection{Robot program in VR}

An online connection between physical robot and the robot in the simulation (Figure

15) can dynamically transmit any movement made in physical space to the robot in the virtual space and vice versa. Thus, avoiding need to have any additional programing. Since the simulation has robot paths in a robot understandable language, the robot program can be transferred back to the simulation to make simulation run according to the physical robot.

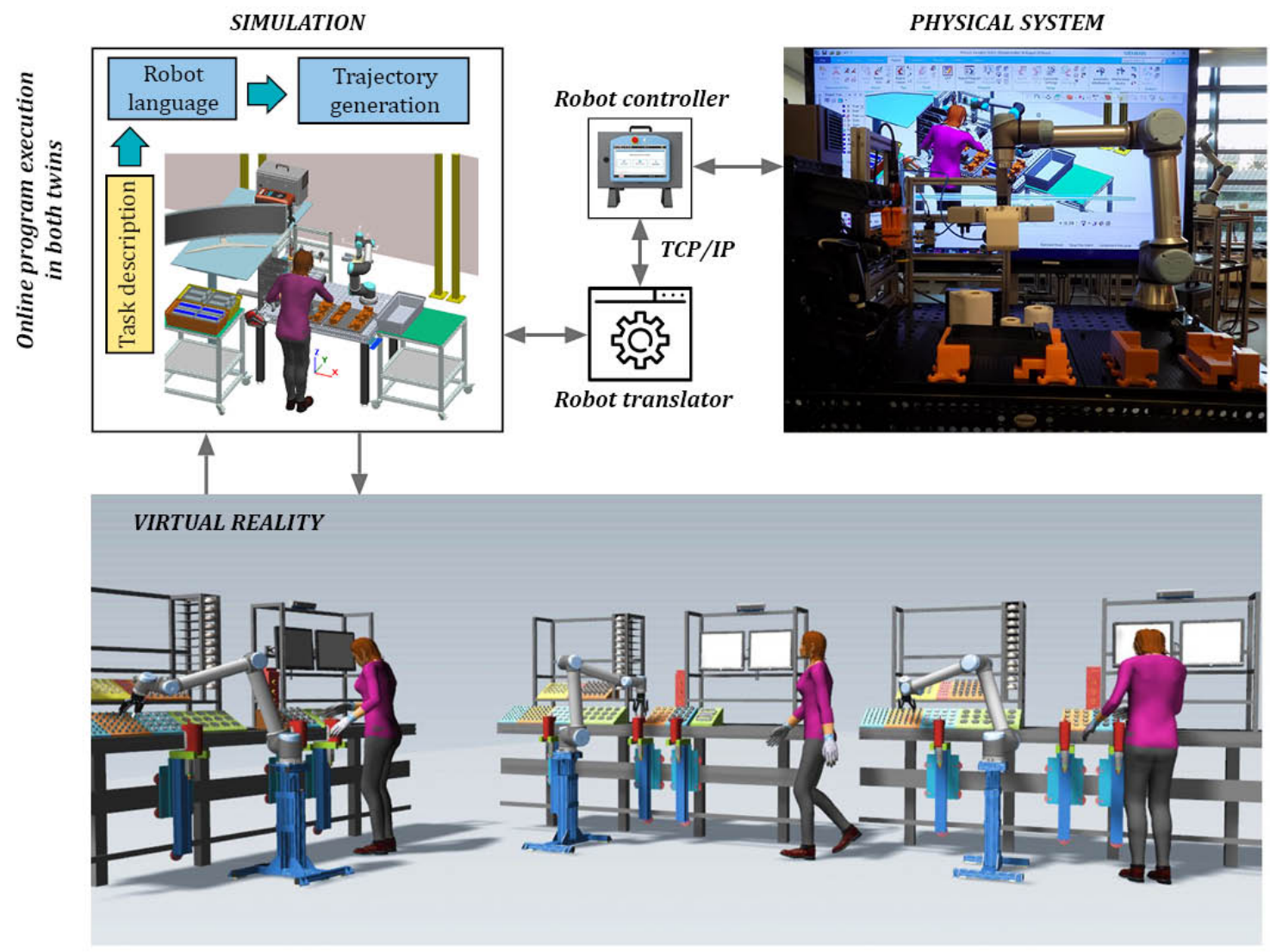

Figure 15: Linking of a real robot, simulation and VR 
In this environment, the VR can be generated online. The user in VR can drag the robot EOT to the pick point and the key location is defined in the simulation. Similarly, remaining points are stored in the simulation, and a simulation cycle is generated. That is transferred to the robot for task execution. Another benefit of this method is that the robot can be visualized in a larger factory setup. And the user in the lab can experience the scene in a larger context.

\subsection{Virtual chatbot as VR assistant}

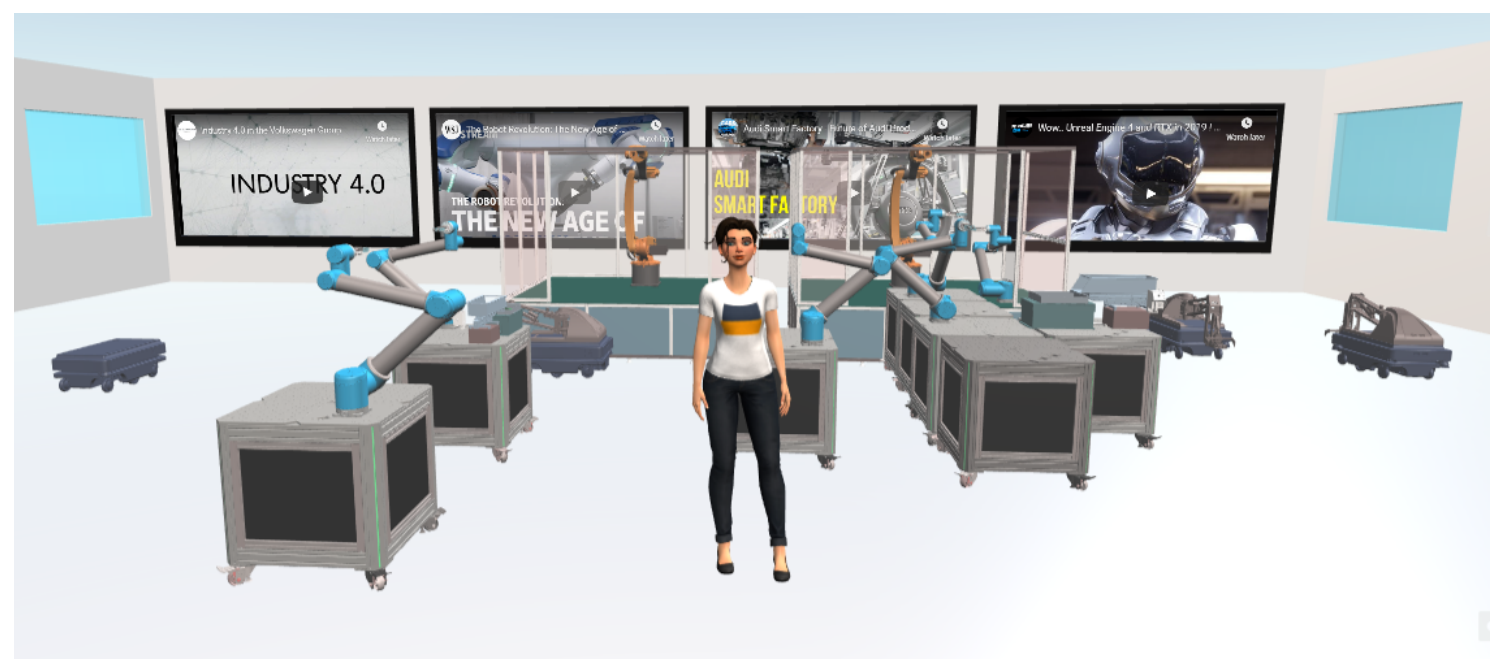

Figure 16: Virtual chatbot in the VR environment based on Amazon AWS

Additionally, a VR environment is developed using Amazon Sumerian (Figure 16). The purpose of this environment is to link the machine state data (robot and the assembly station) connected over the cloud as IoT devices. Amazon Sumerian is used to create the $3 \mathrm{D}$ virtual scene where a soft robot (virtual human character) is used that is linked with the state-machine and can read the performance messages being communicated to the chatbot. The VR environment can be viewable by multiple users at the same time where a virtual human assistant helps the users (as host). Human chatbots are based on Amazon Lex technology which are embedded with IoT cloud data linked to 
the real-time performance of a robot or the whole system (Figure $17 \& 18$ ). Making it a rich and a realistic VR experience for day-to-day planning in future smart factories.

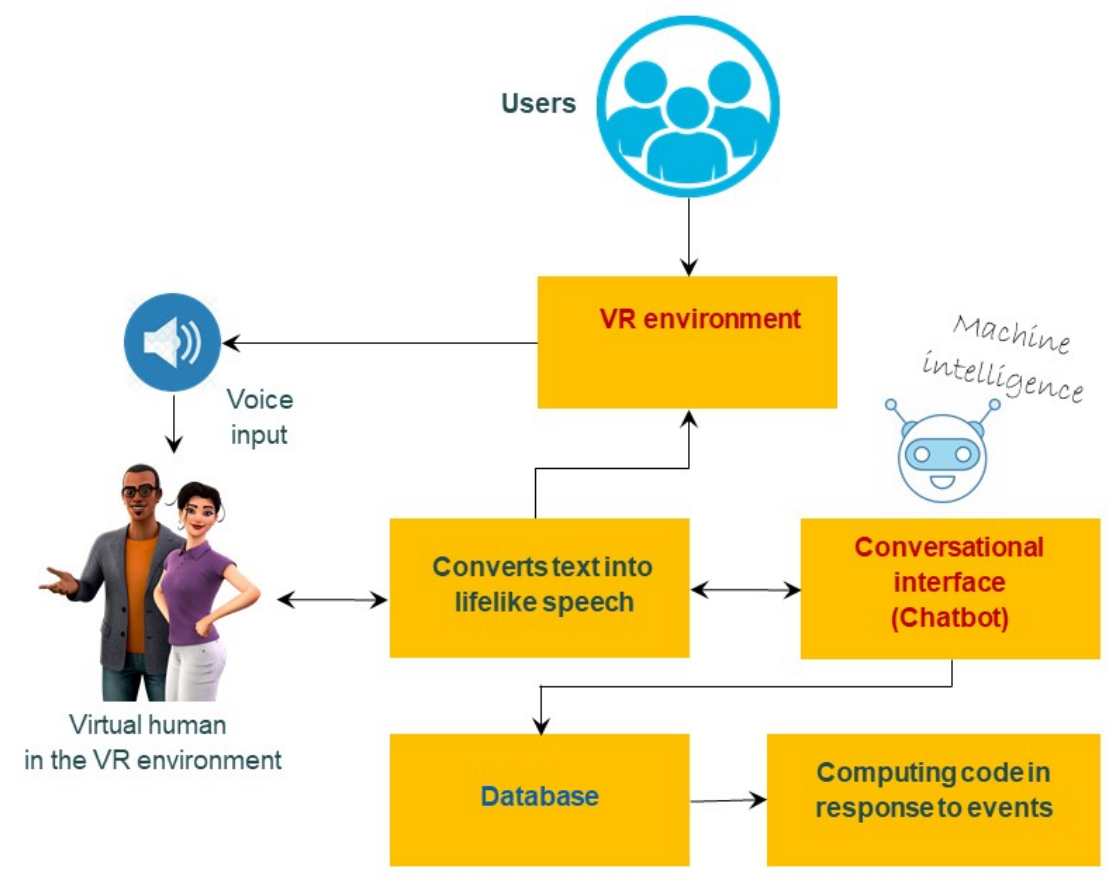

Figure 17: Intelligent virtual human assistant in the VR environment

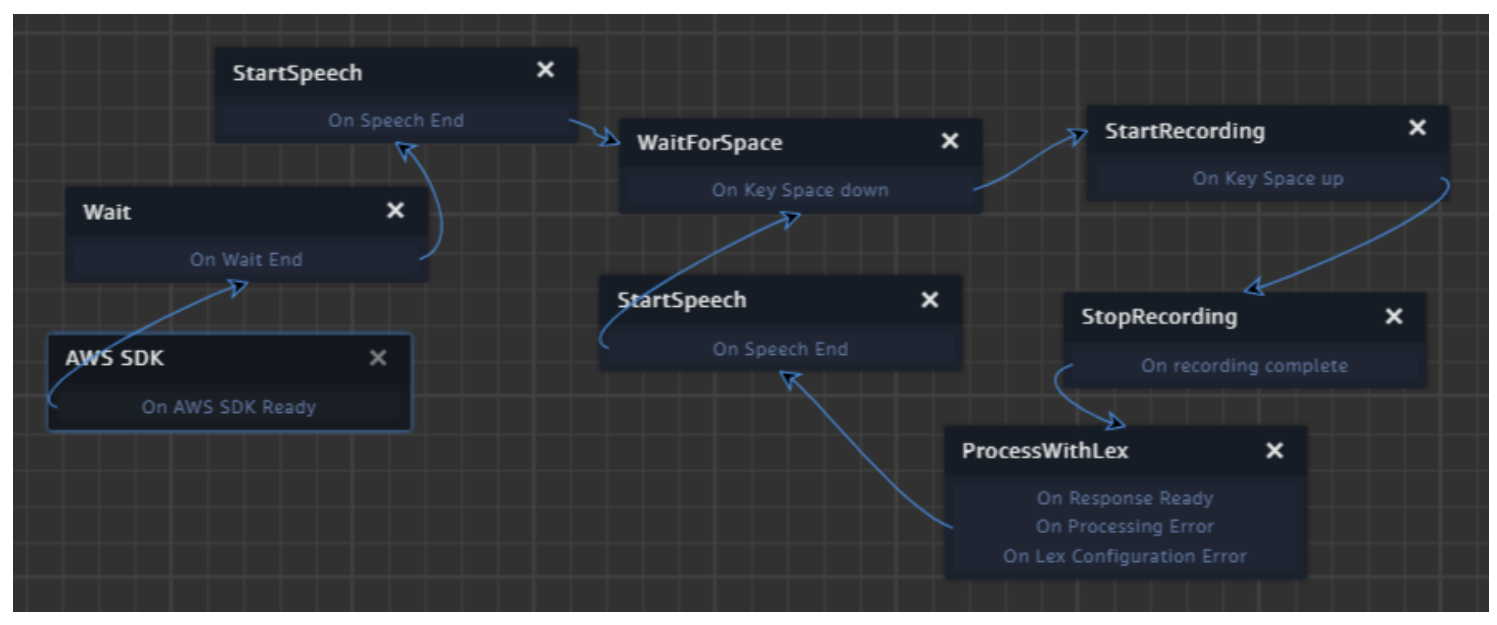

Figure 18: Speech behavior of the virtual-human

\section{Discussion}

From the literature and industrial practices, it is evident that VR has been recognized as a tool to investigate the behaviour of complicated or complex system. A 3D interactive and immersive VR can help acquiring maximum information than from a 
two-dimensional computer screen. Although it is hard to define the cost of extracting this information but for any given process if the cost of information is less than wasting resources (time, energy and material) then the information must be traded-off (Grieves 2014). Since human-robot systems are complex and the errors identified at the later stage can be expansive and fatal due to interaction of a human with a machine, it is justifiable to acquire maximum information during the design phase.

The development in VR is comparable to the technological development of engineering design from paper-based drawings to 3D static computer models to 3D dynamic simulations, and now interactive virtual models. Each wave of technologies for extracting the information was offering a new level of understanding for the designers to develop better designs with new analysis which previously were impossible to make. Just as an example, with 2D drawings making mass property analysis was not possible but with $3 \mathrm{D}$ data it is possible to make mass properties analysis, dynamic interference analysis and many others.

The study demonstrated the usefulness of event-based human-robot simulation linked with VR. The structured framework helped to define the steps and the stages when simulation and VR are helpful in the HRC design process. This simplifies for the design managers to better devise a VR strategy at the organization level. Additionally, the VR is linked with a real robot for faster programming. The demonstration is made for an industrial case and the demonstrator was tested with the workers (end-users) of the cobot based assembly cell. The demonstrator helped them to understand the future production strategy.

Although, the paper presents a few scenarios where the virtual reality can support the HRC design process however, the focus is on discussing the capabilities that modern VR 
tools offer and by doing so the aim is to prompt the readers to think of new applications of virtual simulations integrated with immersive VR.

\section{Conclusion and future work}

The value of HRC systems is apparent for flexibility and higher automation in conventional manual tasks but associated challenges are keeping the technology away from complex and dexterous HRC industrial operations. New methods of designing and validating human-robot work systems are needed for safe and fast embed into mainstream production. The use of simulation, as a risk-free space, in design of complex systems is not new however the advancement in visualization technologies is elevating its usefulness. The paper discussed how the simulation and virtual reality of visualization, immersion, and hapticity can be used to design and evaluate a human robot production system. A framework is presented for simulation and VR based validation of HRC.

With the help of the case study the framework for HRC design is demonstrated for its reliability and feasibility. The tests helped to better visualize the workstation, optimize the simulation by virtually entering the scene and developing a safer workstation in less time. The proposed framework also simplifies the robot programming by reducing the number of steps required (as the programming is achieved as a by-product of validating the virtual assembly station).

The robot programming approach via VR as discussed by the authors is a possibility to explore further. It is intuitive, it is easier to program the robot with humangestures in a virtual environment but the process is still not industry ready. Another limitation is the use of hand-held controllers which doesn't allow to interact with the assets in the VR scene with natural hand movements. It is also a hindrance for performing assembly tasks by a human together with a virtual robot. Although, such hand tracking 
gloves have been developed and are commercially available, yet the technology is expansive and is hard to access by low budget projects. Nevertheless, VR is a promising technology and with its increasing ability to make maximum sense of artificial reality, it has the potential to alter the life of humans at factory floor.

\section{References}

Agrawal, Amit. 2018. "Putting VR/AR to Work". IEEE Computer Graphics and Applications 38, Nr. 1: 115-118.

Anthes, Christoph, Rubén Jesús Garcia-Hernández, Markus Wiedemann and Dieter Kranzlmüller. 2016. "State of the art of virtual reality technology." Aerospace Conference, 2016 IEEE, 1-19.

Begault, Durand R and Leonard J Trejo. 2000. "3-D sound for virtual reality and multimedia". National Aeronautics and Space Administration. NASA/TM-2000209606.

Bilberg, A. and R. Hadar. 2012. "Adaptable and Reconfigurable LEAN Automation - a competitive solution in the western industry". FAIM 2012 22nd International Conference on Flexible Automation and Intelligent Manufacturing. Helsinki.

Bilberg, Arne and Leo Alting. 1991. "When simulation takes control". Journal of Manufacturing Systems 10, Nr. 3: 179-193.

Bilberg, Arne and Ali Ahmad Malik. 2019. "Digital twin driven human-robot collaborative assembly". CIRP Annals - Manufacturing Technology 68, Nr. 1: 499502.

Billings, Charles E. 1997. "Aviation automation: The search for a human-centered approach". CRC Press.

Brettel, Malte, Niklas Friederichsen, Michael Keller and Marius Rosenberg. 2014. "How virtualization, decentralization and network building change the manufacturing landscape: An industry 4.0 perspective". International Journal of Mechanical, Industrial Science and Engineering 8, Nr. 1: 37-44.

Bar-Cohen, Yoseph. 2003. "Haptic devices for virtual reality, telepresence, and humanassistive robotics". Bio Inspired Intell Robots 122: 73.

Dahl, M, A Albo, J Eriksson, J Pettersson and P Falkman. 2017. "Virtual reality commissioning in production systems preparation". Emerging Technologies and Factory Automation (ETFA), 2017 22nd IEEE International Conference on, 1-7.

Djuric, Ana M, RJ Urbanic and JL Rickli. 2016. "A Framework for Collaborative Robot (CoBot) Integration in Advanced Manufacturing Systems". SAE International Journal of Materials and Manufacturing 9, Nr. 2016-01-0337: 457-464.

Flemisch, Frank, Matthias Heesen, Tobias Hesse, Johann Kelsch, Anna Schieben and Johannes Beller. 2012. "Towards a dynamic balance between humans and automation: authority, ability, responsibility and control in shared and cooperative control situations". Cognition, Technology \& Work 14, Nr. 1: 3-18.

De Giorgio, Andrea, Mario Romero, Mauro Onori and Lihui Wang. 2017. "Humanmachine collaboration in virtual reality for adaptive production engineering". Procedia Manufacturing 11: 1279-1287.

Grieves, Michael. 2014. "Digital twin: manufacturing excellence through virtual factory replication". Florida Institute of Technology - White paper. 
Grieves, Michael W. 2019. "Virtually Intelligent Product Systems: Digital and Physical Twins". Complex Systems Engineering: Theory and Practice, ed. Timothy C, Lieuwen, 256

Hayes, Bradley and Brian Scassellati. 2013. "Challenges in shared-environment humanrobot collaboration". Learning 8, Nr. 9.

Hilfert, Thomas and Markus König. 2016. "Low-cost virtual reality environment for engineering and construction". Visualization in Engineering 4, Nr. 1: 2.

$\mathrm{Hu}$, S Jack, J Ko, L Weyand, HA ElMaraghy, TK Lien, Y Koren, Hl Bley, G Chryssolouris, N Nasr and M Shpitalni. 2011. "Assembly system design and operations for product variety". CIRP Annals-Manufacturing Technology 60, Nr. 2: $715-733$.

Jackson, Mats, Mikael Hedelind, Erik Hellström, Anna Granlund and Niklas Friedler. 2011. "Lean automation: Requirements and solutions for efficient use of robot automation in the Swedish manufacturing industry". International Journal of Engineering Research \& Innovation 3, Nr. 2: 36-43.

Kaluschke, Maximilian, René Weller, Gabriel Zachmann, Luigi Pelliccia, Mario Lorenz, Philipp Klimant, Sebastian Knopp, Johannes PG Atze and Falk Móckel. 2018. "Hipsa virtual reality hip prosthesis implantation simulator". 2018 IEEE Conference on Virtual Reality and 3D User Interfaces (VR), 591-592.

Korves, B and M Loftus. 1999. "The application of immersive virtual reality for layout planning of manufacturing cells". Proceedings of the Institution of Mechanical Engineers, Part B: Journal of Engineering Manufacture 213, Nr. 1: 87-91.

Krüger, Jörg and others. 2017. "Human-centered automation to simplify the path to social and economic sustainability". Sustainable Manufacturing, 85-95. Springer.

Kuehn, Wolfgang. 2006. "Digital factory-integration of simulation enhancing the product and production process towards operative control and optimisation". International Journal of Simulation 7, Nr. 7: 27-39.

Li, Xiao, Wen Yi, Hung-Lin Chi, Xiangyu Wang and Albert PC Chan. 2018. "A critical review of virtual and augmented reality (VR/AR) applications in construction safety". Automation in Construction 86: 150-162.

Liu, Oliver, Daniel Rakita, Bilge Mutlu and Michael Gleicher. 2017. "Understanding human-robot interaction in virtual reality". In: Robot and Human Interactive Communication (RO-MAN), 2017 26th IEEE International Symposium on, 751-757.

Malik, Ali A and Arne Bilberg. 2017. "Framework to implement collaborative robots in manual assembly: a lean automation approach". Proceedings of the 28th DAAAM International Symposium, B. Katalinic (Ed.), Published by DAAAM International, ISSN, 1726-9679.

Malik, Ali Ahmad and Arne Bilberg. 2018. "Digital twins of human robot collaboration in a production setting". Procedia Manufacturing 17: 278-285.

Malik, Ali Ahmad and Arne Bilberg. 2019a. "Human centered Lean automation in assembly". CIRP Manufacturing Systems Conference 2019. Procedia CIRP.

Malik, Ali Ahmad and Arne Bilberg. 2019b. "Complexity-based task allocation in human-robot collaborative assembly". Industrial Robot: the International Journal of Robotics Research and Application 46, Nr. 4: pp. 471-480.

Michalos, George, Konstantinos Kaltsoukalas, Panagiotis Aivaliotis, Platon Sipsas, Andreas Sardelis and George Chryssolouris. 2014. "Design and simulation of assembly systems with mobile robots". CIRP Annals-Manufacturing Technology 63, Nr. 1: 181-184. 
Michalos, George, Anna Karvouniari, Nikolaos Dimitropoulos, Theodoros Togias and Sotiris Makris. 2018. "Workplace analysis and design using virtual reality techniques". CIRP Annals-Manufacturing Technology 67, Nr. 1: pp.141-144.

Michniewicz, Joachim and Gunther Reinhart. 2016. "Cyber-Physical-RoboticsModelling of modular robot cells for automated planning and execution of assembly tasks". Mechatronics 34: 170-180.

Mourtzis, Dimitris, Nikolaos Papakostas, Dimitris Mavrikios, Sotiris Makris and Kosmas Alexopoulos. 2015. "The role of simulation in digital manufacturing: applications and outlook". International journal of computer integrated manufacturing 28, Nr. 1: 3-24.

Mrugalska, Beata and Magdalena K Wyrwicka. 2017. "Towards Lean Production in Industry 4.0". Procedia Engineering 182: 466-473.

Muhanna, Muhanna A. 2015. "Virtual reality and the CAVE: Taxonomy, interaction challenges and research directions". Journal of King Saud University-Computer and Information Sciences 27, Nr. 3: 344-361.

Müller, Rainer, Matthias Vette and Matthias Scholer. 2016. "Robot Workmate: A Trustworthy Coworker for the Continuous Automotive Assembly Line and its Implementation". Procedia CIRP 44: 263-268.

Novák-Marcinvcin, Jozef. 2007. "Theory and practice of virtual manufacturing". Manufacturing Engineering: 85-91.

Ore, Fredrik, Lars Hansson and Magnus Wiktorsson. 2017. "Method for design of humanindustrial robot collaboration workstations". Procedia Manufacturing 11: 4-12.

Pelliccia, Luigi, Marco Schumann, Manuel Dudczig, Michele Lamonaca, Philipp Klimant and Giuseppe Di Gironimo. 2018. "Implementation of tactile sensors on a 3Fingers Robotiq adaptive gripper and visualization in VR using Arduino controller". Procedia CIRP 67: 250-255.

Ranasinghe, Nimesha, Kuan-Yi Lee, Gajan Suthokumar and Ellen Yi-Luen Do. 2014. "The sensation of taste in the future of immersive media". Proceedings of the 2 nd ACM International Workshop on Immersive Media Experiences, 7-12.

Rauschnabel, Philipp A, Alexander Brem and Y Ro. 2015. "Augmented Reality Smart Glasses: Definition, Conceptual Insights, and Managerial Importance". Working paper.

Simon, Sofia C and Tobias Greitemeyer. 2019. "The impact of immersion on the perception of pornography: A virtual reality study". Computers in Human Behavior 93: 141-148.

Takata, Shozo and Takeo Hirano. 2011. "Human and robot allocation method for hybrid assembly systems". CIRP Annals-Manufacturing Technology 60, Nr. 1: 9-12.

Thomas, C, L Stankiewicz, A Grötsch, S Wischniewski, J Deuse and B Kuhlenkötter. 2016. "Intuitive work assistance by reciprocal human-robot interaction in the subject area of direct human-robot collaboration". Procedia CIRP 44: 275-280.

Tsarouchi, Panagiota, Sotiris Makris and George Chryssolouris. 2016. "Human-robot interaction review and challenges on task planning and programming". International Journal of Computer Integrated Manufacturing 29, Nr. 8: 916-931.

Tsarouchi, Panagiota, George Michalos, Sotiris Makris, Thanasis Athanasatos, Konstantinos Dimoulas and George Chryssolouris. 2017. "On a human-robot workplace design and task allocation system". International Journal of Computer Integrated Manufacturing 30, Nr. 12: 1272-1279. 